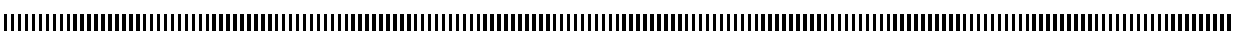
| | | | | |

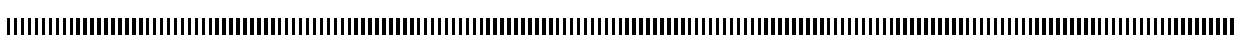

ARIMA

\title{
Solvability of Mixed Problems With Integral Condition for Singular Parabolic Equations
}

\author{
Raid Almomani
}

Math. Department, Yarmouk University, Irbid-Jordan, raid@yu.edu.jo

Department of Mathematics and Natural Scinces, Faculty of Engineering,

Alhosn University, Abu Dhabi, UAE.r.almomani@alhosnu.ae

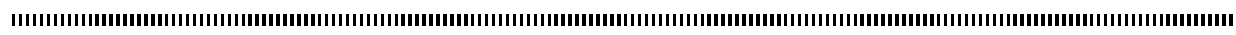

RÉSUMÉ.

ABSTRACT. In this paper we proved the existance and uniqness of strong generalized solution of mixed problems wih integral condition for singular parabolic equaions depending on a theorem proved in [1] in which a priori estimaion of the solution for such problems was derived.

MOTS-CLÉS :

KEYWORDS : Priori estimate; mixed problem; Singular parabolic equations; integral boundary conditions.

2000 Mathematics Subject Classification. 35K20, 35K25, 35K30.

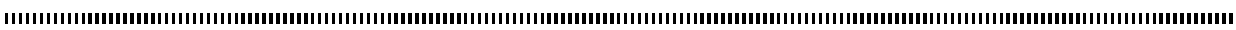




\section{Introduction}

Mixed problems with nonlocal boundary conditions or with nonlocal initial conditions were studied in Bouziani [3], Byszewski et al [4] and [5], Gasymov [7], Ionkin [8]-[9], Lazhar [11], and Said-Nadia [12]. The results and the method used here are a further elaboration of those in [2]. We should mention here that the presence of integral term in the boundary condition can greatly complicate the application of standard functional and numerical techniques. This work can be considered as a continuation of the results in [6] and [13]. $(0, T)$

In [1] the author considered the following mixed problem in the rectangle $Q=(0, l) \times$

$$
\begin{gathered}
L u=\frac{\partial u}{\partial t}-\frac{1}{x^{m}} \frac{\partial}{\partial x}\left(x^{m} \frac{\partial u}{\partial x}\right)=f(x, t), m>0, \\
l u=u(x, 0) \varphi(x),|u(0, t)|<\infty, \iint_{\alpha}^{l} x^{m} u(x, t) d x=0, \alpha>0 .
\end{gathered}
$$

and he proved the following theorem

Theorem $1:$ For any function $u \in E$ such that $x^{\frac{m}{2}} \frac{\partial u}{\partial t} \in L_{2}(Q)$ and $x^{-\frac{m}{2}} \frac{\partial}{\partial x} x^{m} \frac{\partial u}{\partial x}$ $\in L_{2}(Q)$, the following inequality holds

$$
\|u\|_{E}^{2} \leq c\|\mathcal{F}\|_{F}^{2},
$$

where $c=2\left(l+\exp \left(\frac{T}{2 \alpha^{2}}\right)\right)$.

\section{The Main Result}

we consider the operator $L=(\mathcal{L}, l)$ with the following domain

$$
D(L)=\left\{u \in E: x^{\frac{m}{2}} \frac{\partial u}{\partial t} \in L_{2}(Q), x^{\frac{m}{2}} \frac{\partial}{\partial x}\left(x^{m} \frac{\partial u}{\partial x}\right) \in L_{2}(Q)\right\},
$$

acting from $E$ into $F$ by the rule $L u=(\mathcal{L} u, u(x, 0))$.

In a standard way its proved [10] that the operator is closable which we denote by $\bar{L}$ with the domain $D(\bar{L})$.

Definition : The solution of the equation $\bar{L} u=\mathcal{F}$ is called strong generalized solution of the problem (1)-(2). In other words, the function $u$ is called strong generalized solution of the problem (1)-(2) if there exist a sequence of functions $u_{n} \in D(L)$, such that the $\left\|u_{n}-u\right\|_{E} \rightarrow 0$ and $\left\|L u_{n}-\mathcal{F}\right\|_{F} \rightarrow 0$ at $n \rightarrow \infty$.

Theorem 2 : Strong generalized solution of the problem (1)-(2) exist and unique for any $\mathcal{F}=(f, \varphi) \in F$.

Proof. For the sequence $u_{n} \in D(L)$ the following inequality holds

$$
\left\|u_{n}\right\|_{E}^{2} \leq 2\left(l+\exp \left(\frac{T}{2 \alpha^{2}}\right)\right)\left\|L u_{n}\right\|_{F}^{2},
$$


which implies from theorem 1. Passing in (4) to the limit at $n \rightarrow \infty$, we get the following inequality

$$
\left\|u_{n}\right\|_{E}^{2} \leq 2\left(l+\exp \left(\frac{T}{2 \alpha^{2}}\right)\right)\left\|\bar{L} u_{n}\right\|_{F}^{2}, u \in D(\bar{L}) .
$$

From (5) implies that strong generalized solution of problem (1)-(2) is unique, the range $R(\bar{L})$ of the operator $\bar{L}$ is closed in $F$ and $R(\bar{L})=\overline{R(L)}$. Therefore for the proof of existence of strong generalized solution of (1)-(2) we need to prove that the range $R(L)$ of the operator $L$ is dense in $F$.

Since the range of the operator $L$ is dense in a space with the norm $\left(\iint_{0}^{l} x^{n}\left(\varphi^{\prime}\right)^{2} d x+\frac{m}{l-\alpha} \iint_{\alpha}^{l} x^{m-1} \varphi^{2} d x\right)^{\frac{1}{2}}$, its sufficient to show that the equality

$$
\iint_{Q} x^{m} \mathcal{L} u v d x d t=0, \forall u \in D_{0}(L)=\{u \in D(L): u(x, 0)=0\}, x^{\frac{m}{2}} v \in L_{2}(Q),
$$

imply the equality $v=0$.

We set in (6) $x^{m} v=M h$, where $M h=x^{m} h$ at $0 \leq x \leq \alpha$ and

$$
M h=\frac{l-x}{l-\alpha} x^{m} h(x, t)+\frac{1}{l-\alpha} \iint_{\alpha}^{x} \xi^{m} h(\xi, t) d \xi
$$

at $\alpha \leq x \leq l$. This holds if the function $h(x, t)=v(x, t)$ at $0 \leq x \leq \alpha$ and

$$
h(x, t)=v(x, t)-\frac{1}{(l-\alpha) x^{m}} \int_{\alpha}^{l} \int^{m} v(\xi, t) d \xi,
$$

at $\alpha \leq x \leq l$. It is not hard to see that the function $h$ satisfy the third condition from (2), that is

$$
\iint_{\alpha}^{l} x^{m} h(x, t) d x=0 .
$$

So for any function $u \in D_{0}(L)$ and given function $h$ we get the equality

$$
\iint_{Q} \frac{\partial u}{\partial t} M h d x d t=\iint_{Q} \frac{1}{x^{m}} \frac{\partial}{\partial x}\left(x^{m} \frac{\partial u}{\partial x}\right) M h d x d t .
$$

We set in (9) $u=\iint_{0}^{t} w(x, r) d r$ where $w$ is any function such that $x^{\frac{m}{2}} w \in L_{2}(Q)$,

$$
\frac{1}{x^{\frac{m}{2}}} \frac{\partial}{\partial x}\left(x^{m} \frac{\partial w}{\partial x}\right) \in L_{2}(Q), \int_{\alpha}^{l} \int^{m} w(x, t) d x=0,\left|\frac{\partial}{\partial x} w(0, t)\right|<\infty .
$$

Then we get the equality

$$
\iint_{Q} w M h d x d t=\iint_{Q} \frac{1}{x^{m}} \frac{\partial}{\partial x}\left(x^{m} \frac{\partial w}{\partial x}\right) M g d x d t
$$


where $g(x, t)=\iint_{t}^{T} h(x, \tau) d \tau$. The left side of (10) show that the map

$$
L_{2}(Q) \ni x^{\frac{m}{2}} w \rightarrow \iint_{Q} \frac{1}{x^{m}} \frac{\partial}{\partial x}\left(x^{m} \frac{\partial w}{\partial x}\right) M g d x d t \in R
$$

is linear continuos functional. Consequently

$$
x^{\frac{m}{2}} \frac{\partial}{\partial x} \frac{M g}{x^{m}} \in L_{2}(Q), \frac{1}{x^{\frac{m}{2}}} \frac{\partial}{\partial x}\left(x^{m} \frac{\partial}{\partial x}\left(\frac{M g}{x^{m}}\right)\right) \in L_{2}(Q),
$$

and by virtue of (5)

$$
x^{\frac{m}{2}} \frac{\partial}{\partial x} g \in L_{2}(Q), \frac{1}{x^{\frac{m}{2}}} \frac{\partial}{\partial x}\left(x^{m} \frac{\partial}{\partial x} g\right) \in L_{2}(Q),\left|\frac{\partial g(0, t)}{\partial x}\right|<\infty .
$$

Integrating by parts the right hand side of (10) and taking into account (7) and (8), we get

$$
\iint_{Q} w M h d x d t=-\iint_{Q} x^{m} \frac{\partial w}{\partial x} \frac{\partial}{\partial x} \frac{M g}{x^{m}} d x d t
$$

On the basis of (11) we set in (12)

$$
w(x, t)=\iint_{0}^{t} e^{c(\tau-T)} g(x, \tau) d \tau
$$

Then

$$
\iint_{Q} e^{c(t-T)} g M g d x d t=-\iint_{Q} e^{c(\tau-t)} x^{m} \frac{\partial w}{\partial x} \frac{\partial^{2}}{\partial x \partial t}\left(\frac{M w}{x^{m}}\right) d x d t
$$

By analogy of formula (12) in [1] we get

$$
\begin{aligned}
\iint_{Q} e^{c(t-T)} g M g d x d t & =\int_{Q} e^{c(t-T)} \psi(x)|g|^{2} d x d t \\
& +\int_{0}^{T} \int_{\alpha}^{l} \frac{m e^{c(t-T)}}{2(l-\alpha) x^{m+1}}\left|\int_{\alpha}^{x} \xi^{m} g(\xi, t) d \xi\right|^{2} d x d t
\end{aligned}
$$

Further 


$$
\begin{aligned}
\int_{Q} e^{c(T-t)} x^{m} \frac{\partial w}{\partial x} \frac{\partial^{2}}{\partial t \partial x} \frac{M w}{x^{m}} d x d t & =\int_{Q} e^{c(T-t)} x^{m} \psi(x) \frac{\partial w}{\partial x} \frac{\partial^{2} w}{\partial t \partial x} d x d t \\
& -\int_{0}^{T} \int_{\alpha}^{l} \frac{e^{c(T-t)} m}{(l-\alpha) x} \frac{\partial w}{\partial x} \int_{\alpha}^{x} \xi^{m} \frac{\partial w}{\partial t} d \xi d x d t \\
& =\frac{1}{2} \int_{0}^{l} x^{m} \psi(x)\left|\int_{0}^{T} e^{c(t-T)} g(x, t) d t\right|^{2} d x \\
& +\frac{c}{2} \int_{Q} e^{c(T-t)} x^{m} \psi(x)|w(x, t)|^{2} d x d t \\
& -\int_{0}^{T} \int_{\alpha}^{l} \frac{e^{c(T-t)} m}{(l-\alpha) x^{2}} \int_{\alpha}^{x} \xi^{m} \frac{\partial w}{\partial t} d \xi d x d t \\
& +\frac{1}{2} \int_{\alpha}^{l} \frac{x^{m-1} m}{(l-\alpha)}\left|\int_{0}^{T} e^{c(t-T)} g(x, t) d t\right| d x \\
& +\frac{c m}{2} \int_{0}^{T} \int_{\alpha}^{l} \frac{e^{c(T-t)} x^{m-1}}{(l-\alpha)} w^{2} d x d t,
\end{aligned}
$$

where $\psi(x)=\left\{\begin{array}{cc}1, & 0 \leq x \leq \alpha, \\ \frac{l-x}{l-\alpha}, & \alpha \leq x \leq l .\end{array}\right.$

By analogy of (18) in [1] we get

$$
\begin{aligned}
\int_{0}^{T} \int_{\alpha}^{l} \frac{e^{c(T-t)} w}{(l-\alpha) x^{2}} \int_{\alpha}^{x} \xi^{m} \frac{\partial w}{\partial t} d \xi d x d t & \leq \frac{1}{2} \int_{0}^{T} \int_{0}^{l} \frac{e^{c(T-t)}}{(l-\alpha) x^{m+1}}\left|\int_{\alpha}^{x} \xi^{m} \frac{\partial w}{\partial t} d \xi\right|^{2} d x d t \\
& +\frac{1}{2 \alpha^{2}} \int_{0}^{T} \int_{\alpha}^{l} \frac{e^{c(T-t)} x^{m-1}}{(l-\alpha)} w^{2} d x d t
\end{aligned}
$$


From (14) and by virtue of (13),(15)-(17) implies the following inequality

$$
\begin{aligned}
& \int_{Q} e^{c(t-T)} \psi(x)|g|^{2} d x d t+\frac{1}{2} \int_{0}^{l} x^{m} \psi(x)\left|\int_{0}^{T} e^{c(t-T)} g(x, t) d t\right|^{2} d x \\
& +\frac{c}{2} \int_{Q} e^{c(T-t)} x^{m} \psi(x)|w(x, t)|^{2} d x d t+\frac{m}{2} \int_{\alpha}^{l} \frac{x^{m-1}}{(l-\alpha)}\left|\int_{0}^{T} e^{c(t-T)} g(x, t) d t\right| d x \\
& +\frac{m}{2}\left(c-\frac{1}{\alpha^{2}}\right) \int_{0}^{T} \int_{\alpha}^{l} \frac{e^{c(T-t)} x^{m-1}}{(l-\alpha)} w^{2}(x, t) d x d t \leq 0 .
\end{aligned}
$$

We set in (13) $c \geq \frac{1}{\alpha^{2}}$. Then from (18) implies that $g \equiv 0$. Since $x^{m} v=M \frac{\partial g}{\partial t}$ then $v \equiv 0$, and theorem 2 is proved.

\section{Bibliographie}

[1] . Almomani ; A Priori Estimation of the Solution for Mixed Problems with Integral Condition for Singular Parabolic Equations, Mathematical Analysis, Vol. 2014, Article ID 735875, 5 pages, http ://dx.doi.org/10.1155/2014/735875.

[2] . E. Benouar and N. I. Yurchuk; Mixed problem with an integral condition for parabolique equation with the Bessel operator, Differentsial'nye Uravneniya, 27 (1991), p. 2094 - 2098.

[3] . Bouziani ; On the solvability of a class of singular parabolic equations with nonlocal boundary conditions in nonclassical function spaces. IJMMS., $30: 7$ (2002), p. 435 - 447.

[4] . Byszewski ; Existence and uniqueness of solutions of nonlocal problem for hyperbolic equation, J. Appl. Math. Stochastic. Anal.,3 (1990), p. 163-168.

[5] . Byszewski; Differential and functional-differential problems together with nonlocal conditions, Cracow University of technology Monograph, 184, Cracow 1995.

[6] oussa Zakari Djibibe, Kokou Tcharie, Nikolay Iossifovich Yurchuk, Continuous Dependence of Solutions to Mixed Boundary Value Problems for Parabolic Equation, Electronic Journal of Differential Equations, Vol. 2008(2008), No. 17, pp. 1-10.

[7] . A. Gasymov; Mixed problems on the conjugation of parabolic system of different order with nonlocal boundary conditions, Differentsial'nye Uravnenya, 26, No 8, (1991), p. 1003 - 1012.

[8] . I. Ionkin; Solution of boundary value problems in heat conduction theory with nonlocal boundary conditions, Differents. Uravn., $13: 2$ (1977), p. 294 - 304.

[9] . I. Ionkin and E. I. Moiceev; Solution of boundary value problem in heat conduction theory with nonlocal boundary conditions, Differents. Uravn., 13 (1977), p. 294 - 304.

[10] rzysztof Maurin, Methods of Hilbert Spaces, Warszawa, Polish Scientific Publishers, 1967.

[11] . Lazhar; Parabolic equations with nonlocal conditions, Applied Mathematical Sciences, Vol. 1,(2007), No. 21, p. 1041 - 1048.

[12] . Said and L. Nadia; On a nonlocal singular mixed evolution problem, 53 (2001), p. 79 - 89.

[13] . I. Yurchuk; Mixed problem with an integral condition for certain parabolic equations, Differents. Uravn., $22: 12$, (1986), p. 2117 - 2126. 